\title{
Saving Proof from Paradox: Gödel's Paradox and the Inconsistency of Informal Mathematics
}

\author{
Fenner Stanley Tanswell* \\ Arché Philosophical Research Centre, Department of Philosophy, University of St Andrews, \\ St Andrews KY16 9AL, U.K. \\ Department of Philosophy, University of Stirling, Stirling FK9 4AL, U.K.
}

\begin{abstract}
In this paper I shall consider two related avenues of argument that have been used to make the case for the inconsistency of mathematics: firstly, Gödel's paradox which leads to a contradiction within mathematics and, secondly, the incompatibility of completeness and consistency established by Gödel's incompleteness theorems. By bringing in considerations from the philosophy of mathematical practice on informal proofs, I suggest that we should add to the two axes of completeness and consistency a third axis of formality and informality. I use this perspective to respond to the arguments for the inconsistency of mathematics made by Beall and Priest, presenting problems with the assumptions needed concerning formalisation, the unity of informal mathematics and the relation between the formal and informal.
\end{abstract}

Keywords: Informal proof, Gödel's Paradox, Formalisation

\section{Introduction}

Is mathematics consistent? While in practice we generally proceed as if it is, for dialetheists such as Priest in (Priest 1987), mathematics is one of the main battlegrounds on which to establish that inconsistencies do indeed arise and require their dialetheist solutions. In this paper I shall consider two related avenues of argument that have been used to make the case for the inconsistency of mathematics: firstly, paradoxes which lead to contradictions internal to mathematics and, secondly, the incompatibility of completeness and consistency established by Gödel's incompleteness theorems. These two strands of argument are closely connected, for the most apparently problematic paradox in the case of mathematics is Gödel's

${ }^{*}$ This work was supported by the Caroline Elder Scholarship and a St Andrews/Stirling Philosophy Scholarship. Many thanks to two anonymous referees, Aaron Cotnoir, Benedikt Löwe, Noah Friedman-Biglin, Jc Beall, Graham Priest, Alex Yates, Ryo Ito, Morgan Thomas and audiences in St Andrews, Cambridge and Munich. 
paradox, that of the sentence which says of itself that it is unprovable, which is closely related to common constructions of Gödel sentences for formal systems whereby we get to the balancing act between completeness and consistency.

My response to the two lines of dialetheist argument will bring in considerations from the philosophy of mathematical practice on the nature of informal proofs. One thing I will argue for is that we should add to the two axes of completeness and consistency a third axis of formality and informality. Given this third axis, we can consider the dialetheist arguments in two different ways. At the informal end, the previously problematic paradoxes may be genuine, but I argue that there is no compelling reason to see them as internal to mathematics. Meanwhile, at the formal end of the scale, considerations of the practical role of formalisation in mathematics will allow me to make a positive case for incompleteness over inconsistency without begging the question against the dialetheists. My main conclusion will be that the dialetheist arguments considered do not establish that mathematics is inconsistent.

Answering the ultimate question of whether mathematics is consistent from this perspective which encompasses informal proofs and mathematical practice would, I believe, be a major undertaking, and one which I am not intending to complete here. The intention is rather to take the first step in this direction by demonstrating that the matter is not already settled, since the standard arguments from Gödel's theorems and the paradox of provability do not succeed. In fact, I believe these arguments fall apart through a number of the assumptions they need about informal proofs, the nature of mathematics and the process of formalisation, so I shall proceed to raise these objections in turn.

To begin, section 2 will introduce the key distinction between formal and informal proofs that my arguments will focus on. Next, in section 3 I will lay out what Gödel's paradox is and why I do not take it to be a concern for mathematics. In section 4 I lay out Priest's longer argument for the inconsistency of informal mathematics based on the application of Gödel's first incompleteness theorem to informal mathematics and the conclusions he draws from this concerning the inherent inconsistency of informal mathematics. In section 5, I argue that the way of understanding formalisation on which Priest's argument succeeds is a bad one, then show that a better understanding means the argument no longer goes through. In sections 6 and 7, I argue against the thought that we can formalise mathematics as single theory, proposing that a better thought would be to approach formalisation in a fragmented way. Finally, in section $8 \mathrm{I}$ consider formality and informality as a third axis, and a final argument against Priest that he changes the subject in switching between the formal and informal.

\section{Formal and Informal Proofs}

Before we can begin, we need to be sufficiently clear on the distinction between formal and informal proofs, as this will play a central role in the 
remainder of this paper. ${ }^{1}$

Formal proofs are those which are studied in logic and proof theory, and may be defined in the usual way. For example, we might define a formal language, give rules for well-formed formulae in that language, specify axioms to be taken as basic and lay down inference rules for stepping between formulae. A formal proof (relative to such a specified system) will be a (usually finite) sequence of formulae where each is either an axiom or follows stepwise from previous formulae by an application of one of the inference rules, where the final formula is a statement of what was to be proven and is thus established as a theorem in the system.

However, formal proofs are rarely seen in actual mathematical practice. Instead the type of proofs that are employed by mathematicians in their daily activities, teaching and published work tend to be very different. In most cases no formal language is specified, axioms are rarely given and inferences are not confined to just the basic rules. Steps in these proofs can rather be leaps and invoke the background knowledge of your target audience, the semantic understanding of the terms being employed, visualisation, diagrams and topic-specific styles of reasoning. Let us call proofs in this sense informal proofs. Although this would be extremely unsatisfying as a definition, it is certainly not intended as such as one of the main challenges for philosophers of mathematical practice is to pin down exactly what counts as a good, legitimate, correct and rigorous informal proof and filling this out further would take me beyond the scope of this paper. Nonetheless, there is a good deal of literature that does deal with this issue that elaborates on the distinction I am invoking (see Robinson 1991; Hersh 1997; Rav 1999; Leitgeb 2009; Marfori 2010; Larvor 2012 , etc.).

A number of the differences between these two types of proof will affect the assessment of whether the arguments I am considering successfully establish that mathematics is inconsistent. Gödel's first incompleteness theorem relates to proof as an explicitly defined, formal notion attached to a formal system and one of my main counter-arguments in what is to come is that this will not transpose across to apply to informal proofs. Gödel's proof tells us about the limits of formal systems which meet certain conditions, like having a certain amount of expressive power, being able to prove a certain amount of basic mathematics (enough to allow for the required coding etc.) and having an effective procedure for enumerating its theorems. What will be required for the dialetheist line to work, then, will be to show that informal proofs are close enough to formal ones to even begin applying these conditions and that they then meet them. I will argue to the contrary that informal proofs are sufficiently different that the proof will not apply. Some key differences of informal proofs that will play a role later include the social and contextual components of whether such a proof is successful or not; the partially-fragmented nature of modern (informal) mathematics; and the fact that informal mathematics extends to include

\footnotetext{
${ }^{1}$ A terminological note: while I speak of 'informal proofs' and 'formal proofs', some of the literature on this subject instead speaks of 'proofs' and 'derivations' to get at the same distinction. In (Priest 1987), Priest also uses the term 'naïve proof' to refer to the informal proofs.
} 
diagrammatic proofs which have more intuitive inferential rules. Finally, even if the dialetheist arguments manage to establish that informal proofs can be formalised appropriately, there will still be the need to show that the conditions are met. ${ }^{2}$

Before getting the details of the argument from Gödel's theorems, let me assess whether a simpler argument from paradox outlined by Beall is sufficient to show that mathematics is inconsistent.

\section{Gödel's Paradox and Beall's Argument}

The first argument I will consider comes from Gödel's paradox. ${ }^{3}$ Let us begin, therefore, by examining the paradox:

GP: This sentence is (informally) unprovable.

Suppose GP is false; then it is informally provable. Since we take our informal mathematical proofs to establish mathematical truths, it follows that GP is also true. Yet this contradicts the assumption that GP is false, so using proof by contradiction we establish that GP is true. However, since we have just proved GP, it is informally provable. But GP states that it is unprovable, so it must be false. Contradiction.

Now consider how it is that this paradox might show that mathematics is inconsistent. Beall gives the following argument:

There seems to be little hope of denying that $[\mathrm{GP}]$ is indeed a sentence of our informal mathematics. Accordingly, the only way to avoid the above result is to revert to formalising away the inconsistency - a response familiar from the histories of naïve set theory, naïve semantic theory, and so on. If one does this, however, then (by familiar results) one loses completeness, which can be regained only by endorsing inconsistency. Either way, then, we seem to be led to inconsistent mathematics. (Beall 1999, p. 324)

Setting aside the option to formalise away the inconsistency until section 4, the initial argument is that since GP is part of mathematics and GP leads to an inconsistency, it must therefore be that there is an inconsistency in mathematics. In the rest of this section I will undertake the (purportedly hopeless) task of denying that GP is part of mathematics.

The only sensible suggestion as to why GP should be part of mathematics, it would seem, is that GP concerns the broadly mathematical concept of informal provability. I contend, though, that this is not sufficient to make GP a statement of mathematics. The reason is that I take the concept of informal proof to be used to talk and reason about mathematics without it being a part of mathematics. While the former is obvious, for the paradox to render mathematics inconsistent we actually need the

\footnotetext{
${ }^{2}$ In (Priest 1987), Priest argues that these conditions will be met. I believe that the flawed step in the argument is the earlier one of formalisation (as will be covered in section 5), so I will not actively engage in a discussion about whether this formalisation will have an effective calculus etc.

${ }^{3}$ At this point we are only concerned with the informal version of the paradox. Later I take on the formal results of Gödel's theorems.
} 
later, more contentious claim. Of course, I hold that informal proof and provability are very important notions in talking about mathematics, but it is crucial to emphasise that these are notions about mathematics. To establish that the paradox will render mathematics inconsistent, though, we need the extra claim that it is a part of informal mathematics. In general, a statement being about mathematics and a statement being part of mathematics can coincide, but certainly don't always. Consider the following:

(1) Mathematics is traditionally done on blackboards.

(2) This square building with $12 \mathrm{~m}$ sides must have an area of $144 \mathrm{~m}^{2}$.

(3) $111,111,111 \times 111,111,111=12,345,678,987,654,321$.

(4) Ron likes bacon and eggs.

Here (1) is a statement about mathematics but is not itself a part of mathematics. In contrast, (2) is a mathematical statement which is being applied to a situation, so in a relevant sense is not about mathematics. The third item is both mathematical as a statement and about a mathematical fact, while the fourth sentence is neither. Since these two notions can be pulled apart with minimal effort, that a sentence falls under one of them certainly can't constitute a reason to think that it falls under the other. It can therefore be concluded that the notion of informal provability being about mathematics is not sufficient to establish that GP falls within mathematics.

One can also give positive arguments as to why informal provability should not be considered a concept within mathematics. For example, the lack of a precise mathematical definition we observed in section 2 clearly supports the claim that informal provability is not a notion internal to mathematics. Nor does it interrelate with other mathematical concepts in the way that standard mathematical concepts do (such as, for example, group, integer, derivative, line, etc.). The only notable conceptual link it has is with truth, as exploited by the paradox, but if anything the informal notion of truth in mathematics (before being formalised into some formal theory of truth) will belong to the same category of notions about mathematics that are not within mathematics.

By denying that informal provability is a concept within informal mathematics, it can consequently also be denied that GP is a sentence of our informal mathematics. It is thus reasonable to deny that Beall has showed that informal mathematics is inconsistent by using GP. This certainly does not provide an ultimate solution to Gödel's paradox, but it does keep the derived inconsistency out of mathematics and allows us to set aside the paradox to be solved in line with whatever one's favourite solution is to paradoxes generally. ${ }^{4}$

\footnotetext{
${ }^{4}$ A final note on Beall: although the argument I am criticising is from an older paper, the response offered here would fit well with Beall's more recent work in (Beall 2009). The suggestion I have made may be appropriated to make the case that informal proof should join truth in the category of useful devices, which when introduced bring 'merely' semantic paradoxes as by-products or 'spandrels' without thereby rendering the base language (in this case, that of mathematics) inconsistent.
} 
Now, let me note two things about what has gone on here which will be recurrent throughout the paper. Firstly, although this section does not solve Gödel's paradox, this is not really necessary for the purposes of the current project. Beall, Priest and others have a substantial case for the inconsistency of natural languages, a case which is not the target of this paper and would have to be addressed separately if one were so inclined. For both of these authors the claim that mathematics is inconsistent is an additional one that is supported by additional argumentation and it is precisely these arguments which I am targeting. Thus, by rejecting that Gödel's paradox is part of mathematics, what has been done is to show that these additional arguments do not cover more ground than the original case for the inconsistency of natural languages and therefore don't provide added support for dialetheism from the realm of mathematics. Secondly, the separation between being part of mathematics and the concepts used about mathematics is not just a way to re-introduce the object language/meta-language distinction for informal mathematics. A separation of languages is not important because the point is not really one about languages, instead it is about the subject-matter of mathematics. While we may use GP to argue that the concept of informal provability is inconsistent, this does no more work than the liar or any other semantic paradox unless it infects the realm of mathematics. As such, showing that informal proof is not the kind of thing to be investigated mathematically blocks the argument considered in this section.

\section{Priest's Argument for the Inconsistency of Informal Mathematics}

In Chapter 3 of (Priest 1987), entitled "Gödel's Theorem", Priest makes use of Gödel's paradox in the same way as Beall subsequently went on to do, arguing that it shows that informal mathematics is inconsistent. In Priest's case, however, it is given as the culmination of a longer argument which aims to show that informal proof satisfies the conditions for Gödel's first incompleteness theorem in such a way as to lead to its inconsistency. This section will focus on explaining the details of Priest's argument.

Priest wants to show that informal proof is susceptible to Gödel's first incompleteness theorem. The first hurdle is that the theory of informal proofs is, on the surface at least, not formal and hence not immediately susceptible to Gödel's theorem. Priest addresses this in the following way:

It should be said at once that naive proof, or at least the naive theory it generates, is not a formal theory in the sense of the theorem; but it is accepted by mathematicians that informal mathematics could be formalised if there were ever a point to doing so, and the belief seems quite legitimate. The language of naive proof, a fragment of English, could have its syntax tidied up so that it was a formal language, and the set of naïve theorems expressed in this language would be deductively closed. Hence we may, without injustice, talk about the naive theory 
as if it were a formal theory. (Priest 1987, p. 41)

In section 5, I will claim that Priest's reasoning fails to go through at this point. For now, though, let us complete Priest's argument that informal proof satisfies the conditions of Gödel's theorem. The other pieces that Priest needs are that the formalised theory can express all recursive functions and that the proof relation of the formalised theory is recursive. He rightly takes the first requisite to be obviously satisfied and the second to be the contentious one, listing a number of possible objections and his replies. A discussion of these would be irrelevant to the purposes of this paper, so for now we shall grant that the formalised proof relation is recursive.

Given that Priest has now established that informal proof satisfies the conditions of Gödel's theorem, the thrust of his argument is as follows:

For let $T$ be (the formalisation of) our naive proof procedures. Then, since $T$ satisfies the conditions of Gödel's theorem, if $T$ is consistent there is a sentence $\varphi$ which is not provable in $T$, but which we can establish as true by a naive proof, and hence is provable in $T$. The only way out of the problem, other than to accept the contradiction, and thus dialetheism anyway, is to accept the inconsistency of naive proof. So we are forced to admit that our naive proof procedures are inconsistent. But our naive proof procedures just are those methods of deductive argument by which things are established as true. It follows that some contradictions are true; that is, dialetheism is correct. (Priest 1987, p. 44)

Priest soon makes the link between $\varphi$ and Gödel's paradox. For if we take $\varphi$ to be the formalisation of $\mathrm{GP}^{6}$, the inconsistency of section 3 will quickly re-emerge within the formalisation of informal mathematics. A key point is that a standard move towards incompleteness over inconsistency is to separate the object language from the meta-language, but that here we are dealing with informal proof and informal mathematics, for which there is no such distinction, meaning that the orthodox move towards incompleteness is not available. Indeed, this is the entire point of focusing on informal mathematics.

The conclusion that Priest draws is that we are left with true contradictions and dialetheism. ${ }^{7}$ Informal mathematics is seen to be inconsistent, but even more penetratingly he can claim that there is no escape

\footnotetext{
${ }^{5}$ As the target of his argument, Priest needs to explain what he takes naïve or informal mathematics to be exactly. He says:

Proof, as understood by mathematicians (not logicians), is that process of deductive argumentation by which we establish certain mathematical claims to be true. (Priest 1987, p. 40)

His distinction is, in effect, the same as the distinction between formal and informal mathematics as found in section 2 .

${ }^{6}$ The matter is somewhat more complicated than this suggests, of course. Milne discusses in (Milne 2007) the many ways that Gödel sentences can be constructed and what exactly they 'say'.

${ }^{7}$ Not just this, though, since Priest takes it that the theory given by the formalisation of informal mathematics can prove its own soundness and hence must be able to give its own semantics. From here he takes it to follow that it must be able to prove the T-scheme for this
} 
from this application of the incompleteness theorems to informal mathematics and so "... we might say that our naive proof procedures are not just contingently inconsistent, but essentially so... [D]ialetheism is inherent in thought." (Priest 1987, pp. 47-48) That dialetheism is inherent in thought is one of the main claims of In Contradiction, supported by several pillars of argument. The argument described here that informal mathematics is essentially inconsistent forms one of these pillars, but I shall argue that this pillar will not hold any weight.

\section{Formalising Mathematics}

The move from the informal version of mathematics to a formalisation thereof is, in my opinion, too quick. By endorsing the claim that mathematicians take it that informal mathematics can be formalised, Priest moves from the informal theory to the formal one without much consideration of what this move entails or how the mathematicians he is invoking conceive of the formalisation process. For one thing, Priest might not want to endorse the naïve claims of mathematicians at all, since they most likely take mathematics to also be consistent. If such claims were definitive it might thus spell the end of dialetheism.

Nevertheless, it is worth considering how exactly the idea that mathematics should be formalisable will work precisely. In the first half of this section I discuss two options, along with how they interact with Priest's argument. The first follows a straightforward interpretation of Priest's claim but is shown to fail as an account of the formalisation of informal mathematics. The second avoids the problems with the first but, I argue, no longer lets Priest's argument go trough.

\subsection{A First Option}

Let us call the first option many-one formalisation. ${ }^{8}$ The idea is that one takes the entirety of informal mathematics and tidies up the fragment of natural language expressing it to give a formal language. All of the informal theorems will have particular formal counterparts expressed in this one formal language, and the set of these formalised theorems is then deductively closed. For the first option, we consider this as the one single correct formal counterpart for the informal mathematics, a type of super-theory ${ }^{9}$ of mathematics, in which all the current basic assumptions and their consequences are contained. This mirrors a standard idea of formalisation involving a routine procedure of 'filling in the gaps' (as is

theory inside the theory, giving him all of the paradoxes he describes as semantic (as opposed to set-theoretic paradoxes). For example, he lists the liar, Grelling's paradox, Berry's paradox, Richard's paradox and Koenig's paradox as falling under the umbrella of semantic paradoxes. In fact, then, Priest argues that "Our naive theory is semantically closed and inconsistent. By contrast, any consistent theory cannot be semantically closed." (Priest 1987, p. 47)

${ }^{8}$ The 'many' here is due to the fact that it might end up being case that multiple informal proofs are mapped to the same formal proof.

${ }^{9}$ I use the terms 'super-theory' and 'super-system' throughout this paper. I do not intend anything of the 'super-' prefix besides that it is all-encompassing of mathematics in the way described. 
discussed, for instance, in the debate between Rav (Rav 1999, 2007) and Azzouni (Azzouni 2004, 2005) though ultimately rejected by both). Since the formalisation that occurs is crucial to the application of Gödel's first incompleteness theorem to informal mathematics, it would be very convenient for Priest's argument if the picture that is sketched here is the correct one, as this would take formalisation to effectively reduce informal mathematics to something formal, and thereby allow the argument to proceed.

Unfortunately, we have good reason to think that this picture cannot be correct. It is obvious that tidying-up syntax is not going to be a many-one mapping. If we start with the natural-language versions of our mathematical theorems, there will be a whole selection of ways in which we can reproduce these theorems in some particular formal language. Even translating very simple fragments of mathematics into simple formal systems can easily lead to a plurality of results. Scaling this up to include all of mathematics exacerbates this problem significantly. Add to that the fact that we don't start with a particular formal language that we are to be translating the informal into, but instead generate it "on the fly" based on the syntax of our informal mathematics. That there will only be one possible result is clearly absurd. ${ }^{10}$

Note also that the conversion of informal mathematics into this supertheory is not really like the standard conversion of informal mathematics into some 'foundational' theory such as ZFC set theory (which is potentially what the mathematicians that Priest invokes might have in mind). For if this were the case we would quickly find ourselves with the Benacerrafian problem that there are a large number of different adequate representations for our informal concepts (see Benacerraf 1965). This would lead us out of the first option and its super-theory, into a picture where there are multiple different formalisations of informal mathematics.

I would like to emphasise here that the worry I am raising with the generated super-theory is nothing to do with its inconsistency (for such a theory would undoubtedly be inconsistent) and as such it is not open to the usual charge of begging the question against the dialetheist.

\subsection{A Second Option}

As a second option, Priest could hold it that the formalisation process for all mathematics that he is after is actually a case of many-many formalisation. As I have already argued, there may be many different formalisations of mathematics, which Priest can accept as the case in order to avoid the problems presented against the many-one formalisation picture. In essence, this approach is embracing the plurality of formalisations as opposed to letting it become a problem.

However, accepting this path immediately adds an extra complication to the argument, in that now Priest's claims about the formalised ver-

\footnotetext{
${ }^{10} \mathrm{An}$ anonymous referee suggests that we may be able to distinguish between a plurality of results which are equivalent under translation and those which genuinely disagree. I believe, however, that this will not save the argument. In a critical discussion of Azzouni's formalist account of proofs (Tanswell 2015), I have previously argued that such a move is not going to deliver the substantial kind of formalisation required for the argument to proceed.
} 
sion of informal proof must implicitly be quantifying over formalisations. In particular, each time he mentions the formalised version of a proof of informal mathematics, there is no one thing this refers to but instead a selection of different formalised versions of the informal proof. The next natural question to follow this up with is how to determine which formalisations fall under this quantification for any given proof. Put another way: which formalisations of informal mathematics will be adequate and acceptable? For example, a formal language which is too expressively weak to even state standard theorems would be inadequate and unacceptable. The question, then, comes down to finding (and defending) criteria of adequacy for these formalisations of informal mathematics.

Formalisation, as it is being conceived of here, is not a process of exposing an underlying logical form already present in the informal proof, or any thought in this direction. I take this to be the case because informal proofs will underdetermine the language, system and structure that such a proof would adhere to and have. It is instead taken to be a process that is inextricably linked to the context in which it occurs. Relevant factors include the agent performing the formalisation, their purposes in doing so and the formal theory they intend to formalise the given informal proof into. It might be useful here to consider an analogy to Carnap's notion of explication (as in Carnap 1945) where there is also no definitive fact of the matter as to what the correct explication is for some given concept. Instead the different results are compared and evaluated using pragmatic measures such as usefulness, simplicity, explanatoriness, precision etc.

In a similar way, there could be a whole range of formalisations that can be of varying degrees of usefulness in making some informal piece of mathematical reasoning fully formal. In Priest's formalisation of all of informal mathematics we may find a number of different results which are of varying degrees of usefulness, explanation, accuracy, simplicity etc. Of course, amongst these there may be a number of formalisations that we would want to recognise as inadequate, such as that in the above example of an expressively weak language. We want some way of excluding these examples of 'bad' formalisations of informal mathematics from being implicitly quantified over in Priest's argument. However Priest would want to go about this project, we can see that it adds significant philosophical ground that needs to be supplemented to the argument in question before it goes through. ${ }^{11}$

\section{On Mathematical Super-Theories}

A new worry that emerges from the consideration of different formalisations concerns the reliance on one (or indeed many) mathematical supertheories. Since we have seen the analogy to Carnap and want to evaluate our formalisations using pragmatic principles, we must consider whether unified mathematical super-theories, in the sense that Priest has proposed,

\footnotetext{
${ }^{11}$ An anonymous referee proposes an additional argument against Priest based on this section: that the translation on the many-many case is not effective means that informal proof can therefore not meet the minimum requirements for falling under Gödel's theorems. Grist to the mill!
} 
are indeed the best when evaluated in this way. In this subsection I will briefly consider three reasons why this might not be the case.

Before I begin, though, let us just make explicit why for Priest's argument there is now the need to formalise all of informal mathematics in one go, in its entirety, into a super-theory. If this is not done another key step of the argument cannot go through, namely the step where it is insisted that the Gödel sentence is indeed provable. If we were to replay the argument just in arithmetic, for example, we would code in (the formalisation of) informal provability in arithmetic and soon discover the Gödel sentence is not provable in this formalisation. But here we would be free to take the traditional lesson that this is just a limitation on the formalisation, which may well be incomplete. ${ }^{12}$ It is only be squeezing out all room for this incompleteness by quantifying over all mathematics and informal proof simpliciter that the argument could hope to successfully establish that the answer is actually inconsistency rather than mere incompleteness. ${ }^{13}$

Let us now consider why this super-theory will run into difficulties.

One worry may be that different fields or areas of mathematics might be best served by different formal systems, or even different styles of formal systems. For example, the study of algebra, set theory and geometry all appear very different at first glance, and so it may be that they are best served by being formalised into different formal systems (say, with different proof rules which better track the kinds of inferences made in these fields). Of course, the judgment here must be relative to some purpose of formalisation, but we may take the purpose at hand to be (something like) giving a formal reconstruction of the informal proofs, which tracks the inferential steps that were being used. To justify this, recall that Priest's treatment of informal mathematics as a formal theory was meant to be "without injustice".

The first problem I am proposing, then, is that it might be that different formal systems, that are tailored to different sub-areas of mathematics, might allow the more accurate reconstruction of the reasoning present in the informal proofs for those different areas. It also seems that Priest cannot point to the fact that the super-system(s) he is after are those that represent a 'tidying up' of the fragment of natural language that mathematics is expressed in, because the point that is being pressed here is that this talk is an over-simplification of a more complex process.

Relatedly, the second concern I have is that diagrammatic proofs may lead to a significant worry for Priest. In referring to the "fragment of English" that informal mathematics is expressed in, Priest seems to miss a wide selection of mathematics that is communicated pictorially. Pictures can serve to communicate mathematical facts, but can also function as components of informal proofs or proofs in their entirety (see Nelsen 1993, 2000). How is this to be accommodated in the super-systems which are meant to formalise all of informal mathematics? What will the for-

\footnotetext{
${ }^{12}$ And we are well used to theories being incomplete for more reasons than Gödel theorem. For instance, Peano arithmetic also has examples like Goodstein's theorem and the ParisHarrington theorem.

${ }^{13}$ Note that this cannot be avoided by insisting that the Gödel sentence must be part of naïve arithmetic without running afoul of the distinction of section 3 .
} 
malisation process do to diagrammatic proofs? If they are simply to be eliminated, this once again means that informal mathematics is undergoing a drastic change in the formalisation process. Alternatively, there are formal systems for diagrams which may serve to formalise some of the diagrammatic proofs. However, we are now engaged in a project of making the super-systems, which originally sounded straightforwardly close to informal mathematics, encompass much broader pieces of mathematical reasoning. At the very least, this is a non-trivial undertaking which involves constructing a mixed-mode formal system which combines traditional syntactic components with formal diagrammatics. A deeper worry, however, is that we are now able to question whether it will even be possible to capture all of the mathematical reasoning that occurs in informal proofs in formal systems, without doing violence to the source material. I shall return to this line of thought in section 8 .

A third problem we encounter for the mathematical super-theory can draw on Priest's own considerations of mathematical pluralism in (Priest 2012). Modern mathematical investigation extends to examining which results obtain from adopting different logics to work in. Yet if all the various investigations of different logics are taken to be part of informal mathematics, what happens when we formalise them into the one supertheory? Not only do we face the prospect of systems collapsing into one another, but the more alarming danger of triviality looms. Observe that some of the logics we might want to use will include the principle of explosion, most notably classical logic. As soon as a contradiction arises somewhere in the system (which is exactly what Priest's argument is attempting to force), immediately it follows that the whole super-system is trivialised. This is regardless of whether we think that there is something philosophically wrong with classical mathematics, and the principle of explosion in particular, since we are just formalising informal mathematics as we found it. This worry also doesn't rely on logical pluralism, instead just the more uncontroversial fact of logical plurality. ${ }^{14}$ In the case of this worry, Priest's argument will still go through but using the fact that a trivial super-system is also inconsistent, which is hardly a desirable result.

\section{Fragmented Formalisations}

The counter-suggestion to formalising all of informal mathematics simultaneously into one super-theory, with which we have seen some serious difficulties, is that the formalisation process may be one that can only be successful when done in a fragmented way. The suggestion is that constructing a formal system is achievable when we take smaller "chunks" of mathematics that we want to formalise, just not when we want to take it all at the same time. Such an understanding would provide reasonable solutions to dealing with the problems of previous section, without giving up the possibility of formalising parts of mathematical reasoning.

\footnotetext{
${ }^{14}$ I take it that, as mathematicians, we don't need to commit ourselves to the truth, in some philosophical sense, of the mathematics that is being carried out.
} 
Let us see why switching from the idea of a super-theory to the fragmented approach is not a good option if we want to maintain Priest's argument that informal mathematics is inconsistent by Gödel's First Incompleteness Theorem. The issue is that the argument relies on capturing informal mathematics fully to insist that the sentence $\varphi$, which is unprovable in the formalised version of informal mathematics but is nonetheless established by informal proof, must also by provable in the formalised system. If, however, it fails to obtain that any one theory does successfully formally represent all of informal mathematics as a whole, then it cannot be insisted that the last step holds. The point is that we get to the fact that the sentence must be true in the system because the system includes all informal mathematical reasoning. If we do not guarantee this, then the inconsistency is not guaranteed either.

Undermining this last step is sufficient for giving a criticism of Priest's argument, but what we have seen so far forms a somewhat deeper difficulty. Priest's more general project in In Contradiction is to re-examine the balance between completeness and consistency, insisting that it is the latter we jettison in light of Gödel's theorems rather than the former, which is the orthodox choice. Recall that in section 3 we set aside Beall's use of the same balancing act, where he suggests that when formalising mathematical reasoning we are returned to the completeness/consistency dichotomy. What has implicitly been done here, then, is to use considerations of the process of formalisation to give an independent motivation for why we might prefer to end up with an incomplete system when formalising informal proofs, without making reference to any concerns about consistency.

\section{On The Formal and The Informal}

For all that has been said, I think there is another more devastating objection to Priest's argument. In part 5.1 we saw that the idea that there would only be one formalised counterpart of informal mathematics would not hold any water. However, it was only on this reading that it seemed acceptable to treat informal mathematics as if it were a formal theory, at least superficially, stemming from the fact that there was one 'body' of informal mathematics and one formalisation thereof. Nonetheless, having been discussing the difficulties involved in formalising theories, it should now be becoming clearer that there was something fishy going on in this step of the argument.

The objection is the following: by moving from informal proof to a formalised version thereof, Priest's argument is guilty of changing the subject. The argument intended to show that informal proof was inconsistent, and not just coincidentally but inherently so. Yet, almost immediately in the reasoning, to get the application of the incompleteness results off the ground, Priest needs the subject of his argument to be a formal theory. The answer, therefore, is that mathematics is not a formal theory and that transforming it to be one will do an injustice to its source material. The argument speaks as if the multiple representations that informal mathematics can have as formal systems are identical to the 
informal mathematics itself, but this is just a confusion of distinct things.

While Priest was looking to demonstrate that informal mathematics was inherently inconsistent, an option that is now on the table is that mathematical reasoning is inherently informal, a view common in the mathematical practice literature (e.g. Larvor 2012), or that it may be inherently incomplete, or indeed both. The thought would then, in these cases, be that no formal system would suffice to adequately capture mathematics in its entirety. Indeed, this is the traditional lesson that people take from the incompleteness results, but this standard result relies on the question-begging move from consistency to incompleteness. Now, though, we have seen independent motivations for thinking so and rejecting the argument.

Priest's challenge was looking to adjust the balance between consistency and completeness in favour of the latter over the former. But now, by considering the third axis of formality and informality, we have obtained a way to defend incompleteness over inconsistency in the formal setting without begging the question. ${ }^{15}$ For the argument relies on a number of assumptions about the nature of formalisation which allow one to easily and without injustice take informal mathematics into formal mathematics. I have, to the contrary, argued that this distinction runs deep and cannot be bypassed lightly, meaning that arguments that work for formal theories cannot be straightforwardly applied to informal mathematics, and ultimately that Priest's argument does not go through.

\section{References}

Azzouni, J. (2004) "The Derivation-Indicator View of Mathematical Practice", Philosophia Mathematica (III) 12, pp. 81-105.

Azzouni, J. (2005) "How to Nominalize Formalism", Philosophia Mathematica (III) 13, pp. 135-159.

Beall, J., (1999) "From Full Blooded Platonism to Really Full Blooded Platonism", Philosophia Mathematica (III), 7, pp. 322-325.

Beall, J., (2009) Spandrels of Truth, Oxford: Clarendon Press.

Benacerraf, P., (1965) "What Numbers Could Not Be", The Philosophical Review, 74, pp. 47-73.

Carnap, R. (1945) "The Two Concepts of Probability", Philosophy and Phenomenological Research 5, pp. 513-532.

Dutilh Novaes, C., (2011) "The different ways in which logic is (said to be) formal", History and Philosophy of Logic 32, pp. 303-332.

Hersh, R. (1997) "Prove- Once More and Again", Philosophia Mathematica (III) 5, pp. 153-165.

\footnotetext{
${ }^{15} \mathrm{Or}$, at least, to see incomplete and inconsistent systems as both serving purposes which
} may be justified by key pragmatic principles. 
Larvor, B. (2012) "How to think about informal proofs", Synthese 187, pp. $715-730$

Leitgeb, H. (2009) "On Formal and Informal Provability" in Bueno, O. \& Linnebo, Ø. (eds.) (2009): New Waves in Philosophy of Mathematics, Palgrave Macmillan, pp. 263-299

Marfori, M. A. (2010) "Informal Proofs and Mathematical Rigour", Studia Logica 96, pp. 261-272.

Milne, P. (2007) "On Gödel Sentences and What They Say", Philosophia Mathematica (III) 15, pp. 193-226.

Nelsen, R. B. (1993) Proofs Without Words: Exercises in Visual Thinking, Washington: The Mathematical Association of America.

Nelsen, R. B. (2000) Proofs Without Words II: More Exercises in Visual Thinking, Washington: The Mathematical Association of America.

Priest, G., (1987) In Contradiction: A Study of the Transconsistent, Oxford: Oxford University Press.

Priest, G. (2012) "Mathematical Pluralism", Logic Journal of the IGPL, pp. $4-13$.

Rav, Y. (1999) "Why Do We Prove Theorems?", Philosophia Mathematica (III) 7 , pp. 5-41.

Rav, Y. (2007) "A critique of a formalist-mechanist version of the justification of arguments in mathematicians' proof practices", Philosophia Mathematica (III) 15, pp. 291-320.

Robinson, J. A. (1991) "Formal and Informal Proofs" in Boyer, R. S. (ed.) (1991): Automated Reasoning, Kluwer Academic Publishers, pp. 267-282.

Tanswell, F. (2015) "A Problem With The Dependence Of Informal Proofs On Formal Proofs", Philosophia Mathematica (III) 23, pp. 295-310. 